\title{
Brachymetacarpia and Brachymetatarsia in Patients with Multiple Hereditary Exostosis
}

\author{
Yong Jin Cho, MD, Jun Young Lee, MD, Won Gyun Lee, MD*, Sung Taek Jung, MD* \\ Department of Orthopaedic Surgery, Chosun University College of Medicine, Gwangju, \\ ${ }^{*}$ Department of Orthopaedic Surgery, Chonnam National University College of Medicine, Gwangju, Korea
}

Background: Multiple hereditary exostosis is a common autosomal dominant inherited musculoskeletal disorder that manifests with multiple osteochondromas. The clinical manifestations and pathological characteristics of osteochondromas found in the long bone and genetic alterations related to multiple hereditary exostosis have been widely reported. In this study, we investigated the characteristics of brachymetacarpia and brachymetatarsia associated with multiple hereditary exostosis.

Methods: Of the 133 patients with a diagnosis of multiple hereditary exostosis who were recruited from 2005 to 2018, 101 patients who underwent plain radiography after 10 years of age were included. There were 55 male (54.5\%) and 46 female (45.5\%) patients. Brachymetacarpia or brachymetatarsia was diagnosed when disruption of the Lièvre parabola connecting the metacarpal or metatarsal heads was observed on plain radiographs. Three orthopedic surgeons individually reviewed hand and foot plain radiographs.

Results: Of the 101 patients, 41 patients (40.6\%) had more than 1 brachymetacarpia (88 cases) or brachymetatarsia (81 cases). Among 41 cases, $22(53.7 \%)$ were male and $19(46.3 \%)$ were female. The mean age at the time of radiographic evaluation of the hands and feet was 14.6 years (range, 10-63 years). Shortening was most commonly found in the 3rd and 4th metacarpal or metatarsal bones.

Conclusions: We found a relatively high incidence of brachymetacarpia and brachymetatarsia in our patients. Physicians should suspect the presence of brachymetacarpia and brachymetatarsia when treating patients with multiple hereditary exostosis.

Keywords: Multiple hereditary exostosis, Deformity, Bone development, Incidence

Multiple hereditary exostosis (MHE) is a common autosomal dominant inherited musculoskeletal disorder that manifests with multiple osteochondromas and cartilagecapped bony outgrowths at the growth plates. The femur, tibia, and fibula are most commonly affected, but the humerus, radius, ulna, ribs, scapula, and pelvis are also involved. ${ }^{1,2)}$ The number and location of lesions, the degree of orthopedic deformities, and functional impairment vary widely among patients. Approximately $40 \%$ of individuals

Received July 31, 2019; Accepted March 23, 2020

Correspondence to: Sung Taek Jung, MD

Department of Orthopaedic Surgery, Chonnam National University College

of Medicine, 160 Baekseo-ro, Dong-gu, Gwangju 61469, Korea

Tel: +82-62-220-6336, Fax: +82-62-225-7794

E-mail: stjung@chonnam.ac.kr with shortened and/or bowed legs have mild short stature as a result of the deformities. ${ }^{3)}$

We reviewed the literature to identify various physical conditions accompanying MHE and difficulties associated with multiple osteochondromas. Patients with MHE have occasional deformation of the hands and feet, but functional impairment is not severe. There are few reports on the characteristics of the hand or foot of MHE patients. Patients with MHE have hand and foot involvement, but rarely show obvious and specific deformities. The principal area of involvement in the hand appears to be around the metacarpophalangeal joint. Metacarpal shortening usually does not cause functional problems, whereas angular deformity does cause problems and needs surgical treatment. ${ }^{4)}$ Unfortunately, there is no evidence that prevention of deformity is possible by early excision 
of osteochondromas. ${ }^{5)}$ The clinical manifestations and pathological characteristics of osteochondromas found in the long bone in patients with MHE and genetic alterations related to MHE have been widely reported. However, only few studies have focused on the short bone deformities of the hand and foot associated with MHE, and little is known about the characteristics of patients with MHE with regard to the hands and feet. Therefore, we conducted a retrospective radiographic review of patients with MHE of the hands and feet to evaluate the characteristics of brachymetacarpia (BMC) and brachymetatarsia (BMT).

\section{METHODS}

This study was approved by the Institutional Review Board of Chonnam National University Hospital (IRB No. CNUH-2019-091), and the need for informed consent was waived. All procedures were performed in accordance with the relevant policies in South Korea and the tenets of the Declaration of Helsinki. From January 1, 2005, to March 31, 2018, 133 patients with MHE were recruited. MHE was confirmed when at least 2 osteochondromas of the juxta-epiphyseal region of the long bones were observed radiologically. ${ }^{6}$ Of the 133 recruited subjects, 101 patients who underwent plain radiography of both hands and both feet after 10 years of age were included. We were able to proceed with 202 hand and 202 foot plain radiography. $\mathrm{BMC}$ and BMT are described as relative shortening and widening of metacarpal bones in the hands and metatarsal bones in the feet, respectively. BMC or BMT can be diagnosed when disruption of the Lièvre parabola connecting the metacarpal or metatarsal heads is observed on plain radiographs. We used the posteroanterior plain radiographs of the hand to measure the metacarpal length and the dorsoplantar plain radiographs of the foot to measure the metatarsal length (Fig. 1). Three orthopedic surgeons (YJC, WGL, and JYL) individually reviewed the hand and foot plain radiographs (Fig. 2).

We analyzed the incidence of BMC and BMT. The general characteristics and basic data of the patients were summarized using descriptive statistics. The association between the distribution of incidences of BMC and that of BMT was analyzed using the chi-square test. Statistical analyses were conducted using SPSS ver. 18.0 (SPSS Inc., Chicago, IL, USA). A $p$-value $<0.05$ was considered statistically significant.

\section{RESULTS}

Of the 101 patients with MHE, 41 patients (40.6\%) had
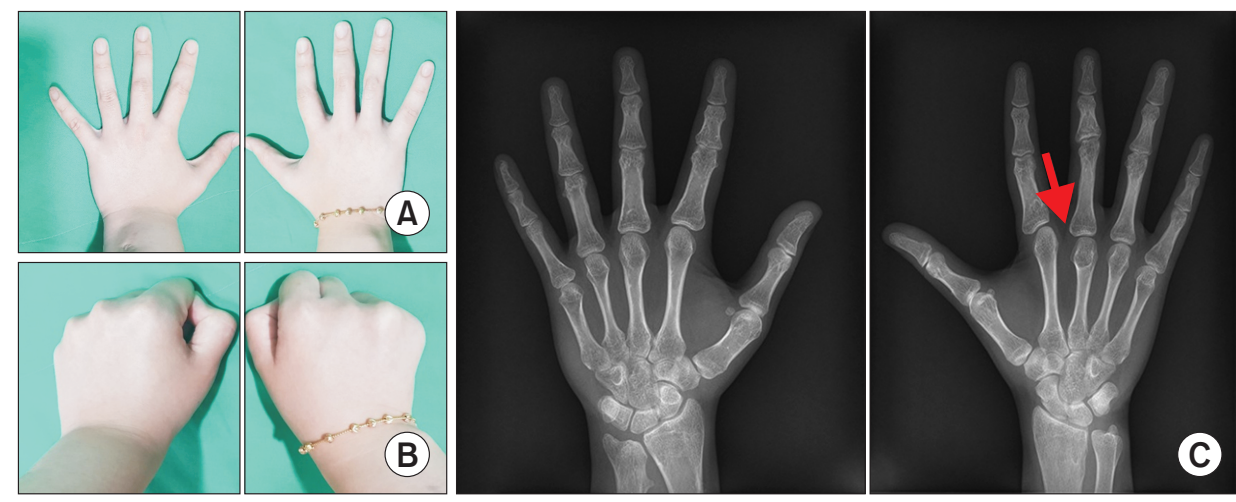

Fig. 1. A 13-year-old female patient with multiple hereditary exostosis underwent follow-up at an outpatient department. (A) The clinical photographs of both hands with fingers spread out did not show parabola disruption. (B) When the patient made a fist, the third metacarpal head looked dented. (C) The right hand radiograph showed a short third metacarpal bone (red arrow).
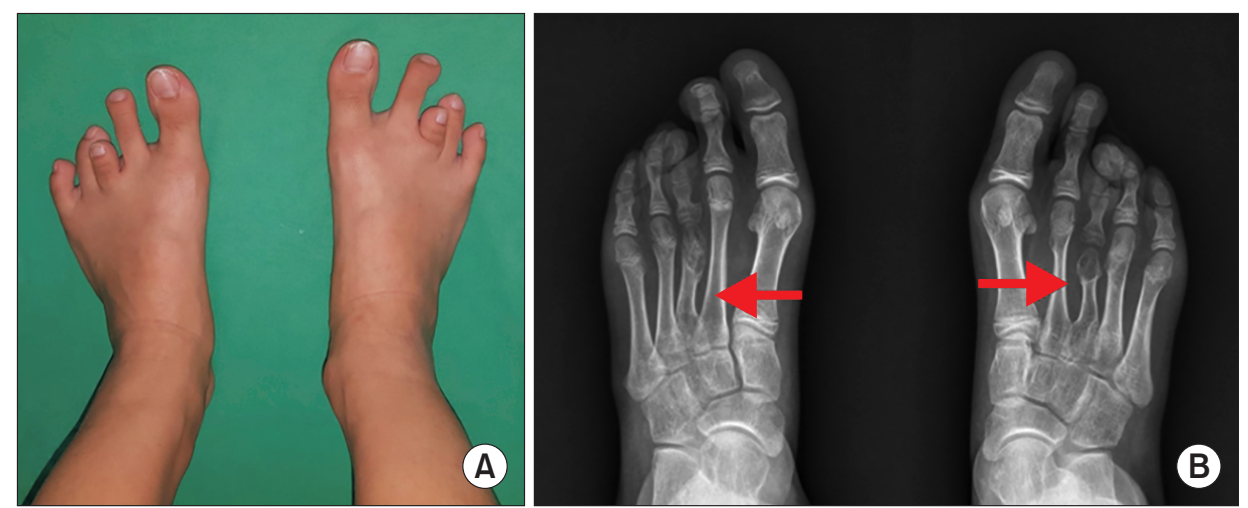

Fig. 2. A 17-year-old male patient with bilateral knee mass and bilateral valgus deformity underwent follow-up radiography at the outpatient department after the diagnosis of multiple hereditary exostosis. (A) Clinical photograph of both feet. (B) Foot radiographs showing the short third metatarsal bones (red arrows). 
Cho et al. Brachymetacarpia and Brachymetatarsia

Clinics in Orthopedic Surgery • Vol. 12, No. 4, $2020 \bullet$ www.ecios.org

more than 1 BMC or BMT. A total of 169 BMCs or BMTs were found in 202 hands and 202 feet. The distributions of BMCs and BMTs for each digit of the hands and feet are shown in Fig. 3. Shortening was most commonly found in the 3rd and 4th metacarpal and metatarsal bones; fewer BMCs were found in the first metacarpal bone and there was no first metatarsal BMT. There was statistically significant difference in the distribution of BMCs and BMTs in each digit of the hand and foot $(p=0.049)$ (Table 1$)$.

Forty-one patients (40.6\%) had more than $1 \mathrm{BMC}$ or BMT. Thirty-six BMCs occurred in the right hand; 31 BMCs, in the left hand; 35 BMTs, in the right foot; and 35 BMTs, in the left foot. Among 41 cases with more than 1 BMC or BMT, $22(53.7 \%)$ were male and $19(46.3 \%)$ were female. The mean age at the time of radiographic evaluation of the hand and foot was 14.6 years (range, 10-63 years).

There were 38 cases (37.6\%) in which at least $1 \mathrm{BMC}$ was found in both hands: 8 cases (7.9\%) had only 1 BMC, 22 cases $(21.8 \%)$ had 2 BMCs, 3 cases (3.0\%) had 3 BMCs, and 5 cases $(5.0 \%)$ had 4 or more BMCs. There were 36 cases $(35.6 \%)$ in which at least $1 \mathrm{BMT}$ was found in both feet: 2 cases (2.0\%) had only 1 BMT, 27 cases (26.7\%) had 2 BMTs, 3 cases (3.0\%) had 3 BMTs, and 4 cases (4.0\%) had 4 or more BMTs.

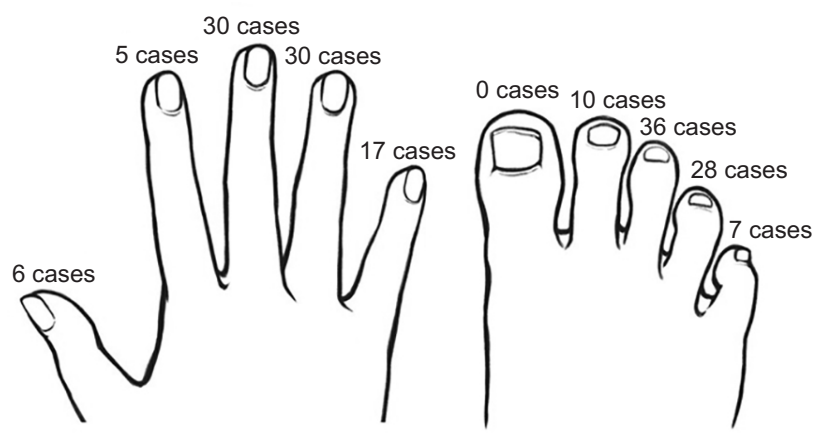

202 hands and 202 feet

Fig. 3. Of the total 101 patients who underwent examination, 41 (40.6\%) had at least 1 brachymetacarpia (BMC) or brachymetatarsia (BMT). Line drawing showing the distribution of BMCs and BMTs for each digit.

\section{DISCUSSION}

MHE is a disease that causes osteochondromas in the long bone physis. However, the metacarpal and metatarsal bones in BMC and BMT are short bones with only 1 physis. This study investigated the abnormal shortness of the metacarpal and metatarsal bones among the short bones of the hand and feet in patients with MHE. Of the total 101 patients included in this study, 5 patients had BMC alone, 3 had BMT alone, and 33 had both BMC and BMT. Sixty of the 101 patients had no BMC or BMT. Over $40 \%$ of the patients with MHE had at least $1 \mathrm{BMC}$ or BMT, which is higher than the previously reported incidences. ${ }^{7-11)}$ This discrepancy can be attributable to differences in the timing of assessment or assessment criteria between reports. In the current study, plain radiographs obtained from patients over 10 years of age were used to improve objectivity of the results. In addition to Turner's syndrome, pseudohypoparathyroidism, cretinism, sickle cell anemia, and MHE should be included as causes of BMC or BMT. ${ }^{12-}$ 16)

In the current study, the number of BMCs and BMTs in MHE patients was greater than the authors had expected. The following hypotheses can be proposed to explain why BMC/BMT was found frequently in the patients with MHE. The first hypothesis is that osteochondromas directly affect the physis and interfere with bone length growth. However, the validity of this hypothesis is questionable in that osteochondromas were not found in all the bones with BMC or BMT and did not necessarily lead to the discovery of BMC or BMT. The second hypothesis is that MHE patients have a pathological physis. Osteochondromas are caused by an abnormality of any component or structure that constitutes the physis, and length growth also interferes with a bone with only 1 physis, such as the metacarpal or metatarsal bone. The third hypothesis is that an unknown chromosomal mutation causes BMC and BMT near the chromosomal mutation that causes MHE. Although the diseases are separate, the distance between chromosome mutations is relatively narrow and the expressions of the diseases often overlap.

Table 1. Comparisons of the Distribution of Brachymetacarpia (BMC) and Brachymetatarsia (BMT)

\begin{tabular}{|ccccccc} 
Case & 1st & 2nd & 3rd & 4th & 5th & Total \\
\hline BMC & 6 & 5 & 30 & 30 & 17 Cases & 81 Cases \\
BMT & 0 & 10 & 36 & 28 & & 7 \\
\hline -value & & & & $0.049^{*}$ & &
\end{tabular}

${ }^{*}$ Chi-square test, statistical significance was considered when $p$-values were $<0.05$. 
Cho et al. Brachymetacarpia and Brachymetatarsia

Clinics in Orthopedic Surgery • Vol. 12, No. 4, $2020 \bullet$ www.ecios.org

Seo et al. ${ }^{3)}$ found over $90 \%$ of patients with MHE were affected by exostoses around the knee and approximately $20 \%$ of the patients had a valgus deformity of the lower extremity and $25 \%$ of the patients were below the 10th percentile for height. Coxa valga, genu valgum, and valgus ankle have been reported as common clinical features of MHE patients. ${ }^{17-19)}$ In our study, hand or foot deformities or both were quite frequent in patients with MHE, but few patients complained of pain or discomfort. The reason for the underestimation of the incidence of BMC and BMT in patients with MHE is probably the more pronounced deformation of the long bone due to the nature of the MHE; the deformation of the hands and feet is generally less noticeable, thus causing less concern in patients. None of the patients with MHE underwent surgical treatment for BMC or BMT. Excision of an osteochondroma was performed on the subject who had difficulty wearing general shoes due to a huge osteochondroma, which was not a treatment performed for BMT but for the osteochondroma.

Hand or foot deformities are difficult to describe on the basis of naked-eye observations. Disruption of the Lièvre parabola connecting the metacarpal or metatarsal heads on anteroposterior plain radiographs and naked-eye observation of disruption of the parabola between the toe tip or fingertip and the tips of the fingers or toes can be the most reliable criteria of BMC or BMT. However, shortening of the first and fifth metacarpal or metatarsal bones may cause the parabola to extend, and then deformation may be ignored or appear almost normal to the naked eye. The reason for the small number of BMCs in the thumb or the absence of BMT in the great toe is unclear because of the difference in the location of the remaining 4 metacarpal or metatarsal bones and physes. Disruption of the Lièvre parabola as a diagnostic criteria may be less sensitive to detect first BMC or BMT. Thus, further studies are needed.

Cates and Burgess ${ }^{16)}$ performed a study that examined 42 hands in 22 MHE patients. They found the fifth metacarpal bone had the highest incidence of exostoses and the distal phalanges and thumb were less affected. Most exostoses were located in the juxta-epiphyseal region. Brachydactyly could be seen in patients with MHE when no exostosis was present. The location and size of the exostosis had no relationship with increased bone shortening. Wood et al. ${ }^{5)}$ reported that the metacarpophalangeal joints of the $3 \mathrm{rd}$ and 4 th fingers were the most commonly affected. In the study of Woodside et al., ${ }^{20)}$ the second and fifth metacarpal bones were the most commonly affected, with the thumb being the least affected. The ulnar side of the hand showed the greatest shortening and angulation. Castes and Wood also identified type B sessile lesions most commonly in the hand, which is similar to the finding reported by Woodside et al. ${ }^{5,16,20)}$

To date, the report by Wood et al. ${ }^{5)}$ is the largest known study on MHE with BMC and BMT based on at presentation and longitudinal data of 99 hands and feet, while another large series includes 83 hands and serial radiographs of 23 patients. ${ }^{20)}$ These studies showed the patients aged 7-10 years had the most tumors per hand, which was related to the rapid skeletal growth during those ages. Cates and Burgess ${ }^{16)}$ reported a transient exostosis rate of $4.9 \%$ in 7 patients based on their longitudinal follow-up data. ${ }^{16)}$ Woodside et al. ${ }^{20)}$ noted a loss of 8 tumors per hand by spontaneous regression and an average gain of 2.7 tumors over 16 years of follow-up.

One of the limitations of this study is that the data were purely radiographic and had no clinical correlation. Shortening was difficult to objectively measure on plain radiographs due to the lack of control of digits. The lack of a strict definition of BMC and BMT (especially for the thumbs or great toes) is a significant limitation of this study.

Patients with MHE had a relatively high incidence of BMT and BMC. The data presented provide physicians with information on the presentation and natural history of MHE through childhood, which would enable them to counsel patients and patients' families on the condition. Physicians should suspect the presence of BMC and BMT when treating patients with MHE.

\section{CONFLICT OF INTEREST}

No potential conflict of interest relevant to this article was reported.

\section{ACKNOWLEDGEMENTS}

An abstract of this work was presented at The 62nd Annual Congress of the Korean Orthopaedic Association 2018.

\section{REFERENCES}

1. Chun YS, Rhyu KH, Cho KY, Cho YJ, Lee CS, Han CS. Osteochondroma arising from anterior inferior iliac spine as a cause of snapping hip. Clin Orthop Surg. 2016;8(1):123-6.

2. Sakamoto A. Usage of a curved chisel when resecting osteo- 
Cho et al. Brachymetacarpia and Brachymetatarsia

Clinics in Orthopedic Surgery • Vol. 12, No. 4, $2020 \bullet$ www.ecios.org

chondroma in the long bone. Clin Orthop Surg. 2013;5(1): 87-8.

3. Seo SG, Sung KH, Chung CY, et al. Incidental findings on knee radiographs in children and adolescents. Clin Orthop Surg. 2014;6(3):305-11.

4. Schmale GA, Conrad EU 3rd, Raskind WH. The natural history of hereditary multiple exostoses. J Bone Joint Surg Am. 1994;76(7):986-92.

5. Wood VE, Molitor C, Mudge MK. Hand involvement in multiple hereditary exostosis. Hand Clin. 1990;6(4):685-92.

6. Bovee JV. Multiple osteochondromas. Orphanet J Rare Dis. 2008;3:3.

7. Tzaveas A, Paraskevas G, Gekas C, Vrettakos A, Antoniou $\mathrm{K}$, Spyridakis I. Anatomical variation of co-existence of 4 th and 5th short metacarpal bones, sesamoid ossicles and exostoses of ulna and radius in the same hand: a case report. Cases J. 2008;1(1):281.

8. Suresh SS, Abraham R, Ravi P. Isolated symmetrical brachymetacarpia of the thumb: case report. Hand (N Y). 2009; 4(4):424-6.

9. Solomon L. Carpal and tarsal exostosis in hereditary multiple exostoses. Clin Radiol. 1967;18(4):412-6.

10. Singh P, Mukherjee SB. Hereditary multiple exostoses, a tale of 50 years. Indian Pediatr. 2015;52(9):795-6.

11. Saglik Y, Altay M, Unal VS, Basarir K, Yildiz Y. Manifestations and management of osteochondromas: a retrospective analy- sis of 382 patients. Acta Orthop Belg. 2006;72(6):748-55.

12. Schimizzi A, Brage M. Brachymetatarsia. Foot Ankle Clin. 2004;9(3):555-70.

13. Page JC, Dockery GL, Vance CE. Brachymetatarsia with brachymesodactyly. J Foot Surg. 1983;22(2):104-7.

14. Matsuno T, Ishida O, Sunagawa T, Ichikawa M, Ikuta Y, Ochi $\mathrm{M}$. Bone lengthening for congenital differences of the hands and digits in children. J Hand Surg Am. 2004;29(4):712-9.

15. Ferrandez L, Yubero J, Usabiaga J, Ramos L. Congenital brachymetatarsia: three cases. Foot Ankle. 1993;14(9):529-33.

16. Cates HE, Burgess RC. Incidence of brachydactyly and hand exostosis in hereditary multiple exostosis. J Hand Surg Am. 1991;16(1):127-32.

17. Clement ND, Porter DE. Can deformity of the knee and longitudinal growth of the leg be predicted in patients with hereditary multiple exostoses? A cross-sectional study. Knee. 2014;21(1):299-303.

18. Clement ND, Duckworth AD, Baker AD, Porter DE. Skeletal growth patterns in hereditary multiple exostoses: a natural history. J Pediatr Orthop B. 2012;21(2):150-4.

19. Denduluri SK, Lu M, Bielski RJ. Development of genu valgum after removal of osteochondromas from the proximal tibia. J Pediatr Orthop B. 2016;25(6):582-6.

20. Woodside JC, Ganey T, Gaston RG. Multiple osteochondroma of the hand: initial and long-term follow-up study. Hand (N Y). 2015;10(4):616-20. 